\title{
An Examination of Hispanic Middle School Students' Interest in Nursing as a Career Choice
}

\author{
Laura McQueen ${ }^{1, *}$, Lynn Zimmerman ${ }^{2}$ \\ ${ }^{1}$ School of Nursing, UNC Chapel Hill, Chapel Hill NC 27514 \\ ${ }^{2}$ Multicultural Education, Purdue University Calumet, Hammond, 46323, Indiana \\ *Corresponding Author: 1lmcquee@email.unc.edu
}

Copyright (C) 2013 Horizon Research Publishing All rights reserved.

\begin{abstract}
This study examines a group of middle school students who are Hispanic and their interest in attending college and pursuing a career in nursing. Although males and females both participated, mostly females expressed an interest in nursing as a profession. In this study investigators examined Hispanic middle school students' knowledge and interest in attending college for a nursing degree and discussed methods to assist in developing more interest in nursing as a career choice for middle school students who are Hispanic. This project supports the need for creating a diverse nursing workforce through inclusion of underrepresented populations. The significance of this study is that it can be used to develop programs aimed at recruitment and retention of student nurses who are Hispanic.
\end{abstract}

Keywords Nursing Career Hispanic Middle School Students

\section{Background}

The healthcare needs of patients who are Hispanic (Hispanic is used throughout this article because it the term used by the US census) have received increased attention in the last decade, with focus on health disparities that effect this population (Askim-Lovseth \& Aldana, 2010). In order to better serve groups culturally that are different from the dominant culture, healthcare must be culturally responsive to patient and caregivers whose culture influences beliefs about health healthcare. In the case of the Hispanic population, cultural responsiveness may also include the ability of healthcare workers to communicate in Spanish. While it is possible to work with this population using interpreters, having healthcare workers who are Hispanic themselves removes some of the possibilities for misunderstanding, miscommunication and distrust. Therefore, one of the issues of concern to the nursing profession is the discrepancy between the size of this rapidly increasing population and the size of the nursing workforce which is Hispanic. The nursing workforce is $2 \%$ Hispanic, whereas the population of the United States who are Hispanic is about $16 \%$ and increasing (US Census, 2012).These numbers reflect a healthcare employment disparity with implications for nursing schools wanting to recruit and retain students from underrepresented groups. Much of the research (Bond, Gray, Baxley, Cason, Denke, \& Moon, 2008; Gilliss, Powell, \& Carter, 2010;Zalaya, Alpert, Xu, \& McDonough, 2011) that has been conducted regarding nursing students who are Hispanic focuses on their high school and college experiences. However, an earlier pilot study conducted by the authors (McQueen \& Zimmerman,2006) as well as a later study by Knight, Abdallah, Findeisen, Melillo, \& Dowling (2011) indicate that middle school is not too early to introduce nursing as a career option.

This project has been partially supported by a grant from the University of North Carolina Program of Ethnicity, Culture, and Health Outcomes (ECHO) which focuses on issues related to racial or ethnic health career disparities, and seeks to determine how best to recruit and retain nursing students who are Hispanic. In this phase of the project, researchers developed, tested, and implemented a needs analysis survey in Guilford and Forsyth Counties in North Carolina for middle school students who are Hispanic about their interested in nursing as a profession. This pilot data was used to uncover Hispanic student's interest in attending college and selecting nursing as an occupation. This initial investigation provides foundational research for developing diverse nursing programs that support the need for culturally competent health professionals. Researchers in this study believe that quality care to underserved populations can be obtained through the realization of successful measures of recruitment and retention of members of target minority populations. Since the Hispanic population is generally underrepresented in nursing, the data collected from middle school students who are Hispanic and who are interested in becoming nurses will be crucial to developing a plan for addressing this disparity in the healthcare workforce. Accordingly, the data from this project could be used to develop programs aimed at the recruitment and retention of nursing students who are Hispanic. 


\section{Theoretical Framework}

Recent studies of eliminating barriers for minorities in health professions has shown that attempts to increase the graduation and integration of the minority nurses in the workforce has not been successful. Barriers identified by NACNEP (2000) in conjunction with the Expert Work groups on Diversity can be categorized as personal and as institutional. Some of the personal barriers that minority nursing students may face include; language issues, financial constraints, inadequate professional career guidance, and inadequate educational preparation. Some of the institutional barriers include the poor imaging of nursing, lack of mentoring programs and minority faculty to attract and nurture minority nurses, and the restrictive admission policies based on standardized tests.

The relatively low numbers of nurses who are Hispanic can be partially attributed to the belief among many Hispanics that college is not a viable option. Existing research (Espinoza-Herold, 2013; Nieto, 2011) suggests that racial, ethnic, cultural, socio-economic, and linguistic biases combine to create a school climate that leads to academic disengagement of many minority students. High school students who are Hispanic become increasingly disengaged from school resulting in their drop-out rate being the highest among all groups (Espinoza-Herold, 2013). According to dropout rate data collected in October 2009 (Child Trends Databank, 2012),39.6\% of all students between the ages of 16 and 24 who drop out of high school are Hispanic. During this same time period $39.2 \%$ of all dropouts were White and $18.1 \%$ are Black. Status dropout rate data, which focuses on background characteristics, such as race/ethnicity, shows that $17.6 \%$ of all students who are Hispanic drop out, while comparison data shows that $5.2 \%$ of all White students and $9.6 \%$ of all Black students drop out.

For those who do complete high school, there are a number of barriers, especially related to cultural and language concerns, which impact Hispanic students' ability to pursue higher education(Espinoza-Herold, 2013; Nieto, 2011). Parents who want their children to go to college may not be able to help them financially. Equally important are issues which prevent these parents from playing an active role in their children's education, such as their own lack of education, their lack of understanding of the American educational system, and their limited English language skills. Another contributor may be the need to help support their families, either through getting a job or by helping with the family business.

\section{Method}

This project is based on the hypothesis: Hispanic students do not see nursing as a career choice, because they lack information on professional nursing and how to pursue a college admission. The goal of this project is to examine the participant's interest in attending college and pursuing a career in nursing.

Prior to implementing this study the researchers received Institutional Review Board approval from the cooperating institutions and acted accordingly with the use of human subjects in research. Survey instruments written in both English and Spanish were also approved.

The researchers in this project recruited the middle school participants for this study from local churches and agencies in the target area because of the difficulty accessing county schools and finding large groups of pre teen Hispanic students. It took several months for the investigators to find the 58 participants, 28 female and 30 males, in the target age of 10-14. The participants self reported as being Hispanic and confirmed they attended schools ranging in grades from 3rd and 9th in the local area. The sample size was limited in size because of the available Hispanic middle school students found in the churches and community agencies frequented in the demographic area studied.

\section{Data Collection}

This project relied on mixed methodology of both quantitative and qualitative data collection and analysis. The two survey instruments developed used a combination of scaled and narrative responses that were written in English and Spanish. The surveys collected demographic information, and then asked questions about their future career plans, knowledge about nursing as a career, general knowledge about college and perceptions of career options, including interest in nursing. Most of the questions required the participants to make a choice, selecting Yes or No, or short response to information, such as, your age or what kind of work would you like to do?

The survey questionnaire had participants identify; plans after high school graduation, desire to be a nurse, family member a nurse, and family member attended college. The variables are represented in the following four questions obtained from this project's questionnaire:

What do you plan to do after high school graduation?

Have you ever thought of being a nurse?

Is anyone in your family a nurse?

Has anyone in your family gone to college?

The four variables were evaluated for frequency and cross-tabulated with one another. In addition, the responses to "Have you ever thought of being a nurse?" were cross-tabulated with gender, identified as the most significant demographic characteristic. The variable reflected in the question "Have you ever thought of being a nurse?" was also cross-tabulated with the nursing characteristics questionnaire.

After administering the questionnaire to the participants, the data from the surveys were analyzed according to the type of data collected. Quantitative data concerning demographics was transferred to Statistical Package for the Social Sciences (SPSS). Frequency charts and cross-tabulations of selected variables were created and 
analyzed for relationships. Using a descriptive research approach the investigators associated and summarized the needs assessment and additional findings that arose out of the project. Correlation studies then examined the relationships among variables, focusing on those students who indicated they had considered nursing as a career.

\section{Findings}

Investigators in this project found that the majority of the 58 respondents, $48(82.8 \%)$ reported a desire to attend college. Thirty-three percent reported that they had thought about being a nurse. The majority of these respondents were female. $(\mathrm{N}=16)$. Another finding identified that 23 (39.6\%) reported a family member had attended college and that 6 $(10.3 \%)$ of the total sample interested in nursing also had a family member who went to college. Additionally, nine of the participants indicated they did not know what kind of work they wanted to do.

The researchers pinpointed 4 significant findings to middle school students' interest in attending college and pursuing a career in nursing. Questions selected from the survey instruments are followed with a discussion of findings:

Finding \#1: What do you plan to do after high school graduation?

Of these 58 participants, $48(82.7 \%)$ said that they definitely would like to go to college when they graduate from high school. The other 10 (17.2\%) marked "job" and/or "I don't know". This data indicates that significant majority of the middle school students who are Hispanic who were surveyed are interested in attending college. This item was marked for further study because a comparison with the literature on college admission rates of students who are Hispanic shows that their admission and retention rates are low compared to other groups.

Finding \#2: Have you ever thought of being a nurse? and Gender?

When the responses to "Have you ever thought of being a nurse?" were cross-tabulated with gender, $57.1 \%$ of the females and $10 \%$ of the males had considered being a nurse. Of the 16 females who said they had thought about being nurses, 5 of them responded that they are planning to be nurses. Of the 3 males who reported they had thought about nursing, none of them chose nursing as a career option, although one said that he would like a job helping people. This item was marked for further analysis, because it supports the existing research that identifies that a preponderance of individuals who choose nursing as a career are women.

Finding \#3: Have you ever thought of being a nurse?" and Is anyone in your family a nurse?

The responses to "Have you ever thought of being a nurse?" were cross tabulated with "Is anyone in your family a nurse?" These results show that being related to a nurse does not seem to affect students' wanting to be a nurse. Of the 19 who said that they had thought about being a nurse, only 2 respondents, $3.4 \%$ of the total sample, also had a family member who was a nurse. This item was marked for further analysis, because it identifies another important area for further study: what does influence someone to become a nurse?

Finding \#4: Have you ever thought of being a nurse? and Has anyone in your family gone to college?

The final finding is the result of the cross-tabulation between the responses from "Have you ever thought of being a nurse?" with the responses from "Has anyone in your family gone to college?" Of the 19 who said that they had thought about being a nurse, 6 respondents, $10.3 \%$ of the total sample who were interested in nursing also had a family member who went to college. This item was marked for further analysis, because literature about college attendance indicates that the mother's education level, in particular, influences a child's motivation to go to college.

\section{Discussion}

In an earlier case study, the researchers on this project (McQueen \& Zimmerman, 2006) found that a sociological framework consisting of 3 levels - the individual/family (micro), community (meso), and institutional (macro) - was useful for analyzing the patterns and connections in the case study's narrative. The analysis indicated that being Hispanic created interconnections among the three levels, the micro level being intertwined with being a member of the Hispanic community (meso) and being a minority in the United States (macro). Similar correlations are evident in the analysis of the data collected from the middle school students in this study.

The findings from this study suggest the following:

Middle school students who are Hispanic are interested in attending college.

Females rather than males who are Hispanic expressed an interest nursing as a career.

This population's desire to become a nurse does not appear to be influenced by a relative who is a nurse.

This population's desire to become a nurse does not appear to be influenced by a relative who has attended college.

Most of the middle school students in this study expressed a desire to attend college. However, the numbers of students who are Hispanic who actually attend college continues to remain proportionately small and underrepresented in baccalaureate education (Espinoza-Herold, 2013; Nieto, 2011). One avenue for further investigation would be to determine what changes occur when middle school students who are Hispanic reach college-age to determine if they still want to go to college and what barriers prevented their attendance. Another question for later research is to examine what influences someone to become a nurse, thereby addressing the limited interest in nursing by middle school students who are Hispanic. 
The significance of this project is that it can be used to develop programs aimed at the recruitment and retention of nursing students who are Hispanic filling a need in the health care field. Based on the data from this project and from the previous study, the researchers propose that schools of nursing will need to provide informational support for students who are Hispanic to assist them in planning for a career in nursing. Interest in college and a career in nursing will not be enough to make this a reality for underrepresented groups in the population. Proactive recruitment efforts that educate these middle school students to the processes of preparing for college, seeking admission, matriculating, and graduating as nurses are key areas of support.

This project proposes two ways to address health disparities as identified by the United States Department of Health and Human Services: (National Goal I), improve access to quality health care through appropriate preparation, composition and distribution of health professions workforce. One step is to increase the recruitment and admission of diverse student populations by analyzing and addressing the needs of students before they begin high school to help them begin preparing early. The second step would be to create and increase an interest in nursing in an underserved population.

Another aim of this project is to address the health care of this target population by increasing the number of nurses that are prepared to care for the complex needs of the economically disadvantaged and underserved in a quality manner (National Goal I), and providing greater access to health care by the education of culturally competent nurses who are able to think critically and problem solve based on priorities to enhance the quality of care (National Goal II).The strength of this research project is that it explores new ground in creating healthcare workforce diversity and awareness of barriers for a career choice in Hispanic population by examining a younger population than previous studies. This research covers an important policy area that provides practical strategies to inclusion and provides needed information to create nursing programs, which will substantially benefit an underserved population. The purpose of the resultant programs based on this study are to provide a more culturally competent health profession workforce to provide quality care to underserved populations, and to increase diversity of the nursing workforce by implementing specific recruitment and retention strategies targeting students who are Hispanic, a minority group that is underrepresented in nursing.

\section{Limitations and Areas for Further Study}

This research project's limitation included; 1) a small sample, 2) language barriers, and 3) a specific geographic location of participants. This project served as a pilot study and had a small sample size with young participants who may have been limited in articulation of their needs and interests' through survey writing. During the research data collection phase, the participants' parents were present to sign for consent to participate. The participants would occasionally defer to their parents for answers to questions, such as, "Who will pay for my college?" or "What is a scholarship?" This observation provided some insight to the researchers on educational information that middle students may be lacking. However, this parental interaction limits the study in social desirability bias (Grimm, 2010). Specifically, the students may have answered not to please the researchers, but to include and please the parents in their decisions.

Another limitation to this project was that the pilot survey did not specify how long the middle school students had lived in and/or attended school in the United States. The surveys were written in both English and Spanish and a nurse who is Hispanic also served as a translator during the data collection phase to answer questions by parents and children. It became apparent during at that time that the participants had varied experiences with language; some could read and answer both Spanish and English, while others could read English or Spanish only, and a few students had to have the questionnaire read to them. The varied educational experiences, grade levels, and English language proficiency of middle school students who are Hispanic suggest that time in the United States is an important element to be included in future research.

An additional limitation included the reliability and validity of the research project. This study focused on a specific sample population and location in the South and did not collect information from Hispanic populations in other regions in the United States. To increase reliability of the data, the study would need to be replicated in other sites with a growing Hispanic population. Responses to this pilot version of the survey instruments also indicate that some of the questions may have resulted in invalid responses because participants did not fully understand the concepts related to the questions. For example, the question "What do you want to be when you grow up?" may have been a more relevant question for this age group than "What kind of work would you like to do?"

\section{Significance and Relevance to Health Disparities}

According to the United States Census Bureau (2011), the population in 2010 in the United States had a 72.4\% White majority. The two largest minority groups were Black, making up $12.6 \%$ of the population, and Hispanic, $16.3 \%$. The US Census Bureau (2012) estimates that by 2060, the percentage of White inhabitants will decrease to around $43 \%$, while the largest minority groups, Black and Hispanic will increase to $15 \%$ and $31 \%$ respectively.

The need for this project and projects like this one is related to not only the nursing shortage that is reaching epidemic proportions nationwide, but also the increased population diversity (US Census Bureau, 2012). This project 
supports the need for creating a diverse nursing workforce through inclusion of underrepresented populations. Researchers in this study examined the participant's interest in attending college for nursing and offered discussion on how to increase the number of Hispanic students to nursing education. The significance of this study is that it can be used to develop programs aimed at recruitment and retention of student nurses who are Hispanic.

\section{Acknowledgment}

Chiu-Yueh Hsiao, PhD, College of Nursing, Chung Shan Medical University, Taiwan, China

\section{REFERENCES}

[1] Askim-Lovseth, M. K., \&Aldana, A. (2010). Looking beyond 'Affordable' Health Care: Cultural understanding and sensitivity-Necessities in addressing the health care disparities of the U.S. Hispanic population. Health Marketing Quarterly, 27(4), 354-387.

[2] Bond, M., Gray, J. B., Baxley, S., Cason, C. L., Denke, L., \& Moon, M. (2008). Voices of Hispanic Students in baccalaureate nursing programs: Are we listening? Nursing Education Perspectives, 29(3), 136-142.

[3] Child Trends Databank. (2012). High school dropout rates: indicators on youth and children. Retrieved from http://www.cfgnh.org/Portals/0/Uploads/Documents/Public/ giveANDlearn-reports/01_Dropout_Rates_0.pdf

[4] Espinoza-Herold, M. (2013).Issues in Latino education: Race, school, culture and the politics of academic success. 2nd Revised ed. Boston: Pearson.

[5] Evans, B. C. (2007). (other article) Application of the caring curriculum to education of Hispanic/Latino and American Indian nursing students. Journal of Nursing Education, 43, 219-228.

[6] Gilliss, C. L., Powell, D. L., \& Carter, B. (2010). Recruiting and retaining a diverse workforce in nursing: From evidence to best practices to policy. Policy, Politics \& Nursing Practice, 11(4), 294-301.

[7] Grimm, P. (2010). Social desirability bias.Wiley
International Encyclopedia of Marketing.[online]. Retrieved from

http://onlinelibrary.wiley.com/doi/10.1002/9781444316568. wiem02057/abstract;jsessionid $=02705864943751$ BAFACA1 5F5A35971E0.d02t04?deniedAccessCustomisedMessage $=\&$ userIsAuthenticated $=$ false

[8] Knight, M., Abdallah, L., Findeisen, M., Melillo, K., \& Dowling, J. (2011). Making healthy connections: Introducing nursing as a career choice to middle school students. Nursing Forum, 46(3), 146-151.

[9] McQueen, L. \& Zimmerman, L. (2006). Using interpretive narrative research methodology in interdisciplinary research projects: Issues in the education of Hispanic Nurses. Journal of Nursing Education. 45 (11), 475-478.

[10] National Advisory Council on Nurse Education and Practice. (2000). National Center for Health Workforce Analysis: The Registered Nurse Population: Findings from the National Sample Survey of Registered Nurses. Retrieved October 1, 2008 from

http://bhpr.hrsa.gov/healthworkforce/data/rnsurvey.htm

[11] Nieto, S. (2011).Affirming diversity: The sociopolitical context of multicultural education 6th ed. Boston: Pearson.

[12] US Census Bureau. (2012). The Hispanic Population in the United States: 2011. Retrieved from http://www.census.gov/ population/hispanic/data/2011.html

[13] US Census Bureau. (2011). Overview of Race and Hispanic Origin: 2010. Retrieved from http://www.census.gov/prod/cen2010/briefs/c2010br-02.pdf

[14] US Census Bureau. (2012). Population by race and Hispanic origin: 2012 and 2060(Percent of total population) [PowerPoint]. Retrieved from http://www.census.gov/newsr oom/cspan/pop_proj/20121214_cspan_popproj_13.pdf

[15] United States Department of Health and Human Services. (2002). 2000 national sample survey of registered nurses. Retrieved March 15, 2008 from http://bhpr.hrsa.gov/healthw orkforce/reports/rnsurvey.htm

[16] United States Department of Education Institute of Educational Services. (2006). Student effort and educational progress. Retrieved May 10, 2008 from http://nces.ed.gov/pr ograms $/$ coe $/ 2007 /$ section3 $/$ table.asp?tableID $=700$

[17] Zalaya, S., Alpert, P. T., Xu, Y., \& McDonough, A. (2011). The Need for Hispanic Nurses in Nevada: An underrepresented ethnic group in the nursing workforce. Home Health Care Management \& Practice, 23(5), 329-335. 